\title{
Ohne Fallgewicht arbeitender Fernauslöser
}

\begin{abstract}
Remote release device operating without messenger

A device is described which can be used where working equipment has to be instantly activated (i.e. without time delay) under water. The latch with an adjustable spring, on which the instrument is fastened, can be released by jerking on the supporting cable or by suddenly stopping the lowering motion, thereby instantly activating the working equipment.
\end{abstract}

Die seit Jahrzehnten gebräuchliche Art, Mechanismen unter Wasser in Bewegung zu setzen, besteht in der Übermittlung des Befehls durch ein Fallgewicht («messenger»), welches beim Aufschlag auf eine vorher gespannte Federeinrichtung oder ein Hebelsystem das betreffende Gerät zum Funktionieren bringt. So werden sozusagen sämtliche Wasserschöpfer mit einem Fallgewicht geschlossen, Umkippthermometer bewegt, Planktonnetze und Sedimentbohrer verschlossen und zum Hieven fertiggemacht usw. In gewissen Fällen wäre es bei solchen Arbeitsgängen erwünscht, den Arbeitsbefehl rascher übermitteln zu können, z. B. bei grossen Wassertiefen. Ein Fallgewicht braucht rund 30 Sekunden für $100 \mathrm{~m}$ Fallhöhe. Diese Zeit, während einer ausgedehnten Probenahme vielfach wiederholt, könnte besser eingesetzt werden. Zudem kommt man immer wieder in die Lage, einen Schliessbefehl direkt, somit ohne zeitliche Verzögerung, ausführen zu lassen, um dadurch einer möglichen Fluchtreaktion von Tieren, z. B. planktischen Copepoden, zuvorzukommen, was für die Repräsentanz von Proben entscheidend sein kann [2].

Wir verwenden seit 1967 den dem Typ «Friedinger» in verstärkter Stahlkonstruktion nachgebildeten Auslöser mit Fallgewicht [1]. Dieses Gerät hat sich in sehr vielen Einsätzen bewährt. Die oben erwähnten Bedingungen für verzögerungsfreie Funktion erfüllt es aber selbstredend nicht. Deshalb wurde aus dem Auslöser, der am 90-1-Wasserschöpfer [3] erfolgreich funktioniert, ein Gerät entwickelt, das, auf beliebige Lasten einstellbar, zwischen das zu aktivierende Arbeitsgerät und das Tragseil eingefügt und durch einen Ruck am Seil ausgelöst wird, worauf die Last freikommt. Es besteht im Prinzip aus einer Klinke, welche durch eine Feder gespannt ist. Diese Feder trägt das Arbeitsgerät. Beim raschen Abbremsen aus der Absenkbewegung oder durch einen Ruck am Seil mit der Handwinde, allenfalls auch durch einen Schlag an das gestoppte Kranseil, wird die momentane Last grösser als die Federspannung, und die Klinke springt auf. Die Funktion geht aus Abb. 1 hervor. Das Gerät ist ganz aus rostfreiem Stahl in handelsüblichen Dimensionen gearbeitet und sehr robust (Abb.2). Es trägt Lasten, welche mit den leichten Hebe-Einrichtungen der limnologischen Feldarbeit (Winden, Kranen) nicht mehr zu bewältigen sind. Es ist klar, dass dieser Fernauslöser die bewährten Fallgewichts- 
systeme nicht ersetzen wird (was auch nicht der Absicht des Autors entspräche), dass er sie jedoch bei besonderen Anwendungen ergänzen kann.

Das Gerät wurde in der Werkstätte der Versuchsstation Tüffenwies der EAWAG hergestellt. Der Verfasser dankt den Herren E. Schwager und Suter für die fachmännische Ausführung.

H. Ambühl
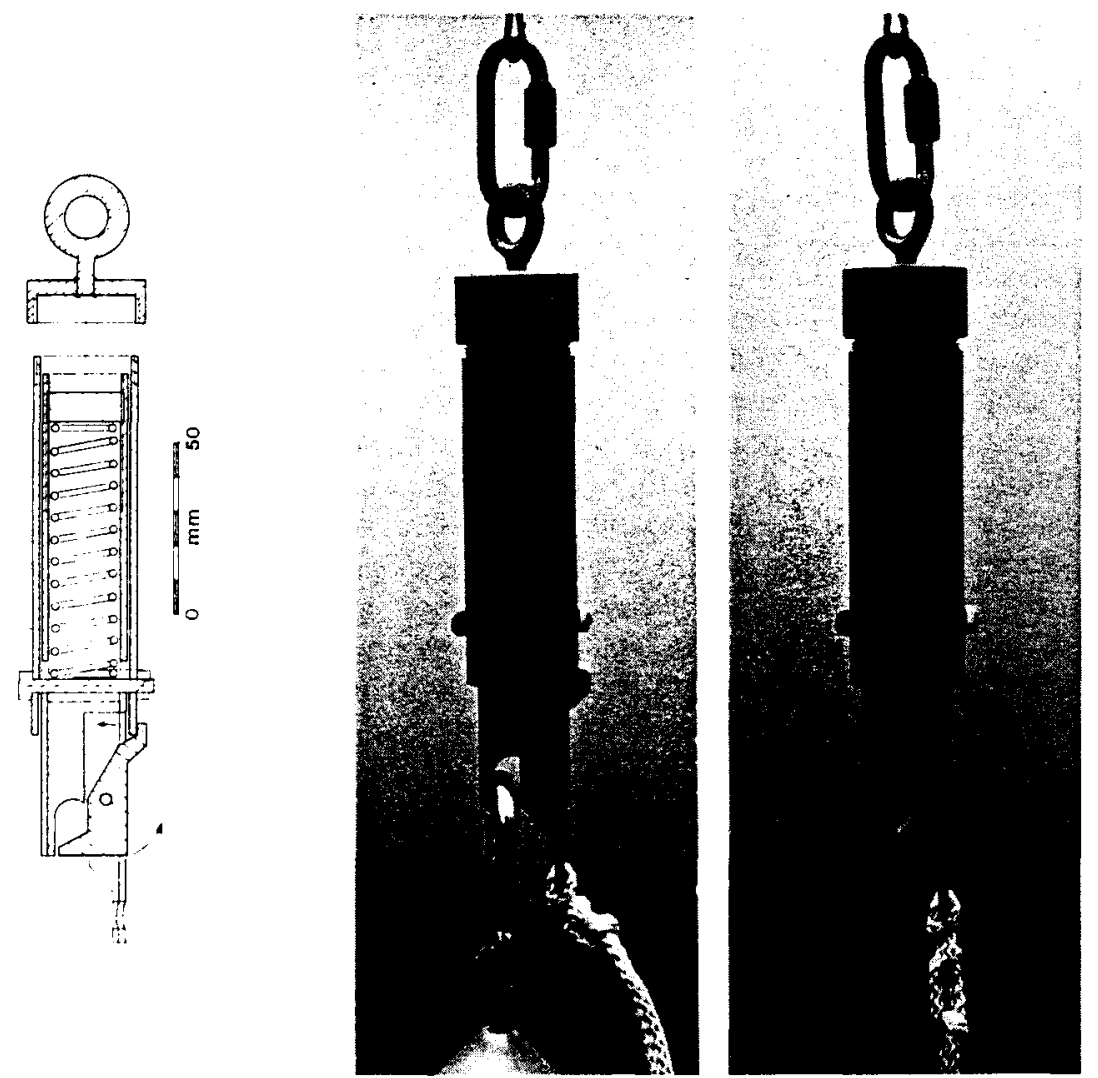

Abb. 1. Konstruktionsskizze. Die Feder presst das innere Rohr nach oben und fixiert dadurch die Klinke. Die Federspannung kann durch die Schraube im inneren Rohr eingestellt werden.

Figure 1. Construction plan. The spring presses the inner piping up and thereby secures the latch. The tension of the spring can be adjusted by the screw in the inner piping.

Abb. 2. Fernauslöser gespannt (links) und ausgelöst (rechts).

Figure 2. Spanned remote releaser (left) and released remote releaser (right). 


\section{LITERATURVERZEICHNIS}

1 Ambühl, H.: Ein Gerät zur Entnahme ungestörter Sediment-Grossproben in Seen. Schweiz. Z. Hydrol. 31, 132 (1969).

2 Bürgi, H.R.: Schweiz. Z. Hydrol. 45 (2), 505 (1983).

3 Ambühl, H.: Ein neuer grossvolumiger Wasserschöpfer für den Fang von Zooplankton. Schweiz. Z. Hydrol. 45 (2), 498 (1983).

Adresse des Autors: Prof. H. Ambühl, EAWAG, CH-8600 Dübendorf, Schweiz. Das Gerät ist erhältlich bei Fa. Dr. H. Züllig, Apparatebau, CH-9424 Rheineck. 